\title{
DIFFERENTIABLE RESTRICTIONS OF REAL FUNCTIONS
}

\author{
JACK B. BROWN
}

(Communicated by R. Daniel Mauldin)

\begin{abstract}
Some new theorems about differentiable, continuously differentiable, or highly differentiable restrictions of continuous and measurable real functions are presented.
\end{abstract}

\section{INTRODUCTION}

When we say that a real function with domain $D$ is differentiable (abbreviated $D^{1}$ ) we mean that $D \subseteq R$ has no isolated points and that the limit

$$
f^{\prime}(x)=\lim _{y \rightarrow x}(f(y)-f(x)) /(y-x)
$$

exists for every $x$ in $D$ (the limit is assumed to be finite and is taken as $y \rightarrow x$ with $y \in D$ ). If $f^{\prime}$ is continuous, we say that $f$ is $C^{1}$. As usual, we say $f$ is $D^{n}$ if it is $n$-times differentiable on $D$, and that $f$ is $C^{n}$ if the $n$th derivative $f^{(n)}$ of $f$ is continuous, and that $f$ is $C^{\infty}$ if $f$ is infinitely differentiable on $D$. If we want to allow $+\infty$ or $-\infty$ as the value of the limit $f^{\prime}(x)$, we say that $f$ is differentiable in the extended sense, abbreviated " $D$ " " with the quotation marks ( $f$ is required to be continuous). If $f$ is " $D^{1}$ " and the extended real-valued function $f^{\prime}$ is continuous in the extended sense, we say that $f$ is " $C^{1}$ ". We say $f^{\prime}$ is continuous in the extended sense if it is continuous in the ordinary sense at each $x$ at which $f^{\prime}(x)$ is finite, and if $f^{\prime}(x)=+\infty$, then for every $B>0$, there exists $\delta>0$ such that for every $y$ in $D$ with $|x-y|<\delta, \quad f^{\prime}(y)>B$ (similar convention if $\left.f^{\prime}(x)=-\infty\right)$. We say that $f$ is " $D^{n}$ " if $f$ is $D^{n-1}$ and $f^{(n-1)}$ is " $D^{1}$ ", and if the extended real-valued function $f^{(n)}$ is continuous in the extended sense, we say that $f$ is " $C^{n}$ ".

Received by the editors February 2, 1989. These results were presented at the 11 th International Summer Symposium on Real Analysis, held in Estergom, Hungary, in August, 1987.

1980 Mathematics Subject Classification (1985 Revision). Primary 26A04, 26A06; Secondary 28A04, 54A03.

Key words and phrases. Differentiable restrictions, $C^{\infty}$, perfect set. 
These properties are of course related as follows:

$$
C^{\infty} \rightarrow \cdots \rightarrow C^{2} \rightarrow D^{2} \rightarrow “ D^{2 ”} \rightarrow C^{1} \rightarrow D^{1} \rightarrow “ D^{1 "} \rightarrow C
$$

The first result of the type we are considering would be the following.

Theorem 1.1. For every continuous $f:[0,1] \rightarrow R$, there exists a perfect subset $P$ of $[0,1]$ such that $f \mid P$ is
(1) monotonic
and

(2) $D^{1}$.

We don't know when this result was first discovered, but it follows from Lebesgue's Differentiability Theorem [14] together with the monotonicity results of Minakshisundaram [10], Padmavally [12], Marcus [9], and Garg [5]. The set $P$ in the conclusion of Theorem 1 cannot be made to have positive measure because of the existence of the almost nowhere approximately differentiable continuous functions of Jarnik [7].

In order to improve the conclusion of Theorem 1 (i.e. to obtain that $f \mid P$ is $C^{1}$ or $D^{2}$ ), it is clear that one needs similar theorems for functions with domains arbitrary perfect sets other than [0,1]. A theorem of this type for monotonic restrictions was established by Filipczak in 1966 [4].

Theorem 1.2. If $P$ is a perfect subset of $[0,1]$, then for every continuous $f: P \rightarrow$ $R$, there exists a perfect subset $Q$ of $P$ such that $f \mid Q$ is monotonic.

Then, a " $D$ " restriction result was established by Bruckner, Ceder, and Weiss in 1969 [3].

Theorem 1.3. If $P$ is a perfect subset of $[0,1]$, then for every continuous $f: P \rightarrow$ $R$, there exists a perfect subset $Q$ of $P$ such that $f \mid Q$ is

(1) monotonic

and

(2) " $D^{1 "}$ ( and "sort of $C^{\infty}$ ").

In order to obtain the "sort of $C^{\infty}$ " conclusion, we have to adopt the convention that if we ever reach the stage where we have a perfect set $Q$ such that $(f \mid Q)^{(n)}$ is identically $+\infty$ (or identically $-\infty$ ), then $(f \mid Q)^{(n+1)}$ exists and is identically 0 .

Morayne gave a simplified proof of Theorem 1.3 in [11].

Theorem 1.1 was drastically improved by Laczkovich in 1984 [8] and a second remarkable result was obtained by Agronsky, Bruckner, Laczkovich, and Preiss in 1985 [1].

Theorem 1.4. If $P$ is a perfect subset of $[0,1]$ and $P$ is of positive measure, then there exists a perfect subset $Q$ of $P$, such that $f \mid Q$ is

(1) monotonic, 
(2) $C^{\infty}$ (relative to $Q$ ) [8], and

(3) extendable to a $C^{1} g:[0,1] \rightarrow R[1]$.

Of course, once you get $f \mid Q D^{1}$, it follows that $f$ can be extended to a $D^{1} g:[0,1] \rightarrow R$, because every $D^{1}$ function with domain a perfect set is so extendable (see [6] or [13]). However, $C^{1}$ functions with perfect domains are not necessarily extendable to $C^{1}$ functions $g:[0,1] \rightarrow R$. In order to obtain (3) of Theorem 1.4, it was necessary to show that the conditions of the "Whitney Extension Theorem" [16] were satisfied.

In $\S I I$, we prove a theorem which essentially adds a condition similar to (3) of Theorem 1.4 to Theorem 1.3 for continuous functions with domains perfect sets. In order to do this, we prove a version of the Whitney Extension Theorem for " $C^{1}$ " extensions. We discuss the example given in [1] which shows that the $C^{1} g$ of Theorem 1.4 (3) cannot be made $D^{2}$, even if you just want an infinite subset $Q$ of $P$. We show by contrast that you can always get an infinite subset $Q$ of $P$ such that $f \mid Q$ is extendable to a " $C^{2}$ " $g:[0,1] \rightarrow R$, even for arbitrary $f$.

In §III, we give a brief discussion of the applications of the above results to measurable functions, present an example, and raise a question which may be of interest to set theorists as well as real analysts.

\section{Continuous FUnctions}

The "Whitney Extension Theorem" [16] is a theorem which gives conditions under which functions $f$ from subsets $D$ of $R^{m}$ into $R^{n}$ are extendable to $C^{k}$ functions $g: R^{m} \rightarrow R^{n}$. The basic case, where $m=n=k=1$ and $D$ is perfect, was used in the proof of part (3) of Theorem 1.4, and the condition in that case is essentially that $f$ be "uniformly differentiable". In order to prove Theorem 2.2, we will need the following " $C^{1}$ " version of Whitney's theorem.

Theorem 2.1. Suppose $P$ is a perfect subset of $[0,1]$ and $f: P \rightarrow R$ is " $D$ ", with $f^{\prime}(x)=+\infty$ for every $x \in P$, and suppose furthermore that for every $B>0$, there exists $\delta>0$ such that if $x$ and $y \in P$ and $0<|x-y|<\delta$, then $(f(x)-f(y)) /(x-y)>B$. Then it follows that $f$ is extendable to a " $C$ " $g:[0,1] \rightarrow R$.

Proof. Extend $f$ as follows. Suppose $(x, y)$ is an open interval contiguous to $P$. Let $S$ be the semicircle with diameter $(y-x) / 3$ which lies above its horizontal diameter, which has left endpoint $(x, f(x))$. Let $T$ be the semicircle with diameter $(y-x) / 3$ which lies below its horizontal diameter, which has right endpoint $(y, f(y))$. Let $L$ be the line which is tangent to $S$ at a point $A$ and tangent to $T$ at a point $B . f$ is extended to $g$ on $[x, y]$ so that $g=S$ between $(x, f(x))$ and $A, g=L$ between $A$ and $B$, and $g=T$ between $B$ and $(y, f(y))$. If $0 \notin P$, then let $y=\min (P)$ and do the same as above with $(x, f(x))=(0,0)$. A similar technique will handle the case where $1 \notin P$. The extension $g$ is clearly " $D^{1}$ " on the complement $P^{c}$ of $P$. We will show that 
$g^{\prime}(x)=+\infty$ if $x \in P$. Let $x \in P$. If $x$ is the endpoint of an open interval in $P^{c}$, the one-sided derivative of $g$ at $x$ from that direction is clearly $+\infty$.

Assume without loss of generality that $x$ is a limit point of $P$ from the right. Suppose $B>0$. Let $\delta>0$ be such that (1) if $u$ and $v$ are elements of $P$ with $0<|u-v|<\delta$, then $(f(u)-f(v)) /(u-v)>1,(2)$ if $v$ is an element of $P$ with $0<|v-x|<\delta$, then $(f(v))-f(x)) /(v-x)>3 B / 2$, and (3) $x+\delta \in P$. Suppose $0<y-x<\delta$. If $y \in P, \quad(f(y)-f(x)) /(y-x)>3 B / 2>B$. If $y \in P$, there are elements $u<v$ of $P$ such that $y \in(u, v) \subseteq P^{c}$. Let $h=f(v)-f(u)$. It is clear from the geometry that every point of $g$ with abscissa in $(u, v)$ lies above the line from $(x, f(x))$ to $(v, f(v)-h / 3)$, which has slope at least $\frac{2}{3}(3 B / 2)=B$. Thus, $(f(y)-f(x)) /(y-x)>B$, and the theorem if proved.

The following is an improvement of Theorem 1.3 which is in the spirit of (3) of Theorem 1.4.

Theorem 2.2. If $P$ is a perfect subset of $[0,1]$, then for every continuous $f: P \rightarrow$ $R$, there exists a perfect subset $Q$ of $P$ such that $f \mid Q$ is extendable to a " $C^{1} " g:[0,1] \rightarrow R$.

Proof. Get the perfect set $Q_{1} \subset P$ such that $f \mid Q_{1}$ is monotonic and " $D^{1}$ ". It follows from Borel measurability of $\left(f \mid Q_{1}\right)^{\prime}$ that there is either a perfect subset $Q_{2}$ of $Q_{1}$ such that $f \mid Q_{2}$ is $D^{1}$ or there is a perfect subset $Q_{2}$ of $Q_{1}$ such that $\left(f \mid Q_{2}\right)^{\prime}$ is identically $+\infty$ (or identically $\left.-\infty\right)$. In the first case, the theorem follows from Lemma 20 of [1]. Assume the second case holds and that $\left(f \mid Q_{2}\right)^{\prime}$ is identically $+\infty$.

As Step 1, pick two points $x_{0}$ and $x_{1}$ of $Q_{2}$ such that $\left(f\left(x_{1}\right)-f\left(x_{0}\right)\right) /$ $\left(x_{1}-x_{0}\right)>1$. Pick disjoint subintervals $I_{0}$ and $I_{1}$ of $[0,1]$ having $x_{0}$ and $x_{1}$, respectively, in their interiors such that if $x \in Q_{2} \cap I_{0}$ and $y \in Q_{2} \cap I_{1}$, then $(f(y)-f(x)) /(y-x)>1$. Pick $\delta_{1}$ to be less than the distance between $I_{0}$ and $I_{1}$.

Suppose $n$ is a positive integer and that $x_{s}$ and $I_{s}$ have been picked for all $n$ term dyadic sequences $s$, and that $\delta_{n}$ has been picked. For each $n$-term dyadic sequence $s$, pick two points $x_{s, 0}$ and $x_{s, 1}$ of $Q_{2} \cap I_{s}$ (interior to $I_{s}$ ) such that $\left(f\left(x_{s, 1}\right)-f\left(x_{s, 0}\right)\right) /\left(x_{s, 1}-x_{s, 0}\right)>n+1$. Pick disjoint subintervals $I_{s, 0}$ and $I_{s, 1}$ of $I_{s}$, having length less than $\delta_{n} / 2$, having $x_{s, 0}$ and $x_{s, 1}$, respectively, in their interiors, and such that if $s \in Q_{2} \cap I_{s, 0}$ and $y \in Q_{2} \cap I_{s, 1}$, then $(f(y)-f(x)) /(y-x)>n+1$. Pick $\delta_{n+1}>0$ to be less than the distance between any two of the intervals in $\left\{I_{s}: s\right.$. is an $(n+1)$-term dyadic sequence $\}$.

$$
Q=\bigcap_{n=1}^{\infty} \cup\left\{I_{s}: s \text { is an } n \text {-term dyadic sequence }\right\}
$$

is a subset of $Q_{2}$ which satisfies the hypothesis of Theorem 2.1, so Theorem 2.2 is proved. 
The following theorem was proved in [1] to show that condition (3) of Theorem 1.4 was sharp.

Theorem 2.3. [1] For every $\in>0$, there exists a perfect subset $P$ of $[0,1]$ of measure at least $1-\in$ and a continuous $f: P \rightarrow R$ such that

(a) $\{x \in P: f(x)=g(x)\}$ is finite for every $D^{2} g:[0,1] \rightarrow R$,

(b) $\{x \in P: f(x)=g(x)\}$ has no bilateral limit point for every " $D^{2}$ " $g$ : $[0,1] \rightarrow R$,

and

(c) $\{x \in P: f(x)=g(x)\}$ has at most finitely many limit points for every “ $D^{2} " g:[0,1] \rightarrow R$.

Actually, only (a) was stated in [1], but it was proved that if $x_{1}, x_{2}, \ldots$ is a monotone decreasing sequence on which $f=g$, and $x$ is the limit of the sequence, then

$$
g^{\prime \prime}(x)=2 \lim _{n \rightarrow \infty}\left(f\left(x_{n},-f(x)\right) /\left(x_{n}-x\right)^{2}=+\infty .\right.
$$

It is also clear that if one considers $x_{1}, x_{2}, \ldots$ to be increasing, it can be argued that $g^{\prime \prime}(x)=-\infty$. Therefore, (b) follows. Furthermore, if each $x_{n}$ is a limit point of the set on which $f=g$, it was shown in [1] that $g^{\prime}(x)=g^{\prime}\left(x_{n}\right)=0$ for each $n$, so that $g^{\prime \prime}(x)= \pm \infty$ would be impossible and (c) follows.

In contrast to Theorem 2.3, the following theorem holds.

Theorem 2.4. If $A$ is a subset of $[0,1]$ of positive outer measure, then for every $f:[0,1] \rightarrow R$, there exists an infinite closed subset $B$ of $A$ such that $f \mid B$ is extendable to a monotonic " $C^{2} " g:[0,1] \rightarrow R$.

Proof. Suppose $A$ and $f$ are as in the hypothesis. It follows from a theorem of Kolmogorov and Vercenko (see Chapter IX of [14]) that there exists an $x \in A$ and a sequence $x_{1}, x_{2}, \ldots$ from $A \backslash\{x\}$ converging to $x$ such that the sequence $\left\{\left(f\left(x_{n}\right)-f(x)\right) /\left(x_{n}-x\right)\right\}$ converges to a finite number $m$. Assume without loss of generality that $x=f(x)=0$ and $\left\{x_{n}\right\}$ is decreasing. Also assume that $\left\{f\left(x_{n}\right) / x_{n}\right\}$ is decreasing and that $m=0$ (we may get monotonicity of $\left\{f\left(x_{n}\right) / x_{n}\right\}$ by taking a subsequence, and we can change to one of the functions $\pm(m t-f(t))$, if necessary). If $\left\{f\left(x_{n}\right) / x_{n}\right\}$ is identically zero, then $\left\{f\left(x_{n}\right)\right\}$ is identically zero, and the theorem follows immediately, so we are assuming that $\left\{f\left(x_{n}\right) / x_{n}\right\}$ is strictly decreasing. By taking a further subsequence, we may also assure that $\left\{f\left(x_{n}\right) / x_{n}^{2}\right\}$ converges to some (possibly infinite) value $m^{\prime}$.

As Case I, assume $m^{\prime}$ is finite. By taking subsequences we could also assure that for each $n$,

$$
\sum_{i=n+1}^{\infty} f\left(x_{i}\right) / x_{i} x_{n}<1 / n \text {. }
$$

Then, by taking further subsequences we can arrange things so that for each $n$

$$
h_{n}=\frac{f\left(x_{n}\right)-f\left(x_{n+1}\right)}{x_{n}-x_{n+1}}
$$


is sufficiently close to $f\left(x_{n}\right) / x_{n}$ so that

(1) $h_{1}, h_{2}, \ldots$ is still decreasing with limit 0 .

(2) $\lim _{n \rightarrow \infty} h_{n} / x_{n}=m^{\prime}$ and

(3) for each $n, \sum_{i=n+1}^{\infty} h_{i} /\left(x_{n}-x_{n+1}\right)<1 / n$.

Define $u:[0,1] \rightarrow R$ so that $u(t)=h_{n}$ if $x_{n+1}<t \leq x_{n}$ and $u(0)=0$. We don't care what happens between $x_{1}$ and 1 , so we will assume for notational convenience that $x_{1}=1$. Notice that if $U(x)=\int_{0}^{x} u(t) d t$, then $U\left(x_{n}\right)=f\left(x_{n}\right)$ for each $n$. However, $U$ is far from " $D^{2}$ " because $u$ is a step function. Now, "jiggle" $u$ by rotating each horizontal piece of $u$ counterclockwise a bit about its center to get the function $v$ defined by

$$
\begin{aligned}
v\left(x_{n}\right)=h_{n}+\left[\left(h_{n}-h_{n+1}\right)-\left(h_{n+1}-h_{n+2}\right)+\left(h_{n+2}-h_{n+3}\right)-\cdots\right] \\
v \text { linear on each }\left[x_{n+1}, x_{n}\right], \text { and } v(0)=0 .
\end{aligned}
$$

It can be checked that the endpoints match up, so that $v$ is continuous and if $V(x)=\int_{0}^{x} v(t) d t$, we still have $V\left(x_{n}\right)=f\left(x_{n}\right)$ for each $n$. Furthermore, since

$$
\frac{2 h_{n}}{x_{n}} \geq \frac{v\left(x_{n}\right)}{x_{n}} \geq \frac{2 h_{n}}{x_{n}}-2\left[\frac{h_{n+1}-h_{n+2}+\cdots}{x_{n}}\right]
$$

it follows from (3) that $\lim _{n \rightarrow \infty} v\left(x_{n}\right) / x_{n}=2 m^{\prime}$. Since $v$ is linear on each $\left[x_{n+1}, x_{n}\right]$, it follows that $v^{\prime}(0)=2 m^{\prime} . v^{\prime}$ is constant on each $\left(x_{n+1}, x_{n}\right)$ and has value

$$
\frac{2 h_{n}}{x_{n}-x_{n+1}}-4\left[\frac{h_{n+1}-h_{n+2}+\cdots}{x_{n}-x_{n+1}}\right] \text {, }
$$

so it follows from (3) that $\lim _{t \rightarrow 0} v^{\prime}(t)=2 m^{\prime}\left(v^{\prime}\right.$ does not exist at $\left.x_{1}, x_{2}, \ldots\right)$.

Now we "smooth out the corners" on $v$. At each $x_{n}(n \geq 2)$, consider an $x_{n+1}<a<x_{n}<b<x_{n-1}$ where $(b-a)<\frac{1}{3} \min \left(x_{n+1}-x_{n}, x_{n}-x_{n-1}\right)$ and $c=x_{n}=(a+b) / 2$. Let $\in=\left|v^{\prime}(a)-v^{\prime}(b)\right|$. We may replace $v \mid[a, c]$ by a $C^{1}$ function $q:[a, c] \rightarrow R$ such that

(4) $q(a)=v(a), q(c)=v(c)$,

(5) $q^{\prime}(a)=v^{\prime}(a), q^{\prime}(c)=v^{\prime}(b)$,

(6) $\int_{a}^{c} q(t) d t=\int_{a}^{c} v(t) d t$, and

(7) $\left|q^{\prime}(t)-v^{\prime}(t)\right|<2 \in$ for every $t \in[a, c)$.

Now, let $w$ be the function which consists of all such replacements $q$ together with the part of $v$ which is not replaced, and let $g(t)=\int_{0}^{t} w(x) d x$. Equations (4) and (5) make $w C^{1}$ on $(0,1]$, and (7) makes $\lim _{t \rightarrow 0} w^{\prime}(t)=$ $\lim _{t \rightarrow 0} v^{\prime}(t)=2 m^{\prime}$, so $w$ is actually $C^{1}$ on $[0,1]$, and $g$ is $C^{2}$ on $[0,1]$ in this case. Equations (4) and (6) keep $g\left(x_{n}\right)=f\left(x_{n}\right)$ for every $n$, and it is clear that $g$ is monotonic.

As Case II, assume $m^{\prime}=+\infty$. Proceed exactly as in Case I, and at the end we will have $w$ continuous on $[0,1], C^{1}$ on $(0,1]$, and (7) will make $\lim _{t \rightarrow 0} w^{\prime}(t)=\lim _{t \rightarrow 0} v^{\prime}(0)=+\infty$. So $w$ is " $C^{1}$ " on $[0,1]$ and $g$ is " $C^{2}$ " on $[0,1]$ and monotonic, and the theorem is proved. 
Note 2.5. In the above proof, we assumed that $x_{1}, x_{2}, \ldots$ was decreasing. It actually follows from the theorem of Kolmogorov and Vercenko that $x_{1}, x_{2}, \ldots$ can be made to be increasing or decreasing (at your choice). Thus, the set where $f=g$ (for a " $C^{2} " g$ ) can be made to have as many limit points from the left and as many limit points from the right as you want, as long as you only want finitely many of each and you don't want bilateral limit points.

\section{Measurable FUnCtions}

Variations on Lusin's theorem for functions measurable with respect to various $\sigma$-algebras were considered in [2]. The $\sigma$-algebras of interest were $L$ (the Lebesgue measurable sets), $U$ (the universally measurable sets), $B$ (the Borel measurable sets), $(s)$ (the Marczewski measurable sets), $B_{w}$ (the sets with the Baire property in the wide sense), and $B_{r}$ (the sets with the Baire property in the restricted sense). See [2] for the appropriate definitions. These $\sigma$-algebras are related as follows:

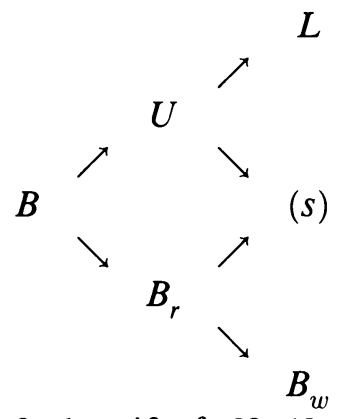

It follows from Theorem 2.2 that if $f:[0,1] \rightarrow R$ is $L-,(s)-$, or $B_{w^{-}}$ measurable, then there exists a perfect subset $Q$ of $[0,1]$ such that $f \mid Q$ is extendable to a " $C^{1} " g:[0,1] \rightarrow R$. It follows from Theorem 4.1 of [3] that if $f$ is $U$ - or $B_{r}$-measurable, then every perfect subset $P$ of $[0,1]$ has a perfect subset $Q$ such that $f \mid Q$ is extendable to a " $C^{1}$ " $g:[0,1] \rightarrow R$. In fact, $(s)$-measurability characterizes the functions $f$ for which this is true.

It is also clear from Lusin's Theorem that if $f:[0,1] \rightarrow R$ is $L$-measurable then there exists a perfect subset $Q$ of $[0,1]$ such that the stronger conclusions of Theorem 1.4 hold. By contrast consider the following.

Example 3.1. There exists a $B_{w}$-measurable $f:[0,1] \rightarrow R$ with no $D^{1}$ restriction to any perfect subset of $[0,1]$.

Proof. Let $g:[0,1] \rightarrow R$ be a strictly increasing continuous function with $g(0)=0, \quad g(1)=1$, and such that $g^{\prime}(x)=+\infty$ at each element $x$ of a dense $G_{\delta}$ subset $M$ of $[0,1]$. Let $h:[0,1] \rightarrow[2,3]$ be a "Sierpinski-Zygmund [15] function" which has no continuous restriction to any set of cardinality $c$. Let $f(x)=g(x)$ if $x \in M$, and let $f(x)=h(x)$ if $x \in M^{c} . f$ clearly satisfies the requirement.

Problem 3.2. Does there exist a $B_{r}$-measurable (or even an $(s)$-measurable) $f:[0,1] \rightarrow R$ which has no $D^{1}$ restriction to any perfect subset of $[0,1] ?$ It is 
shown in Example 2 of [2] that $C H$ implies the existence of a $B_{r}$-measurable $f:[0,1] \rightarrow R$ which has no continuous restriction to any set of positive outer measure, but we do not see how to use this to answer this question.

\section{REFERENCES}

1. S. Agronsky, A. M. Bruckner, M. Laczkovich and D. Preiss, Convexity conditions and intersections with smooth functions, Trans. Amer. Math. Soc. 289 (1985), 659-677.

2. J. B. Brown and K. Prikry, Variations on Lusin's theorem, Trans. Amer. Math. Soc. 302 (1987), 77-86.

3. A. M. Bruckner, J. G. Ceder and M. L. Weiss, On the differentiability structure of real functions, Trans. Amer. Math. Soc. 142 (1969), 1-13.

4. F. Filipczak, Sur les fonctions continues relativement monotones, Fund. Math. LVIII (1966), 75-87.

5. K. M. Garg, On level sets of a continuous nowhere monotone function, Fund. Math. LII (1963), 60-68.

6. V. Jarník, Sur l'extension du domaine de définition des fonctions d'une variable qui laisse intacte la dérivabilité de la fonction, Bull. Int. Acad. Sci Bohême, (1923).

7. __ Sur les nombres dérivés approximatifs, Fund. Math. 22 (1934), 4-16.

8. M. Laczkovich, Differentiable restrictions of continuous functions, Acta Math. Hungar. 44 (1984), 355-360.

9. S. Marcus, Sur les fonctions continues qui ne sont monotones en acun intervalle, Rev. Math. Pures Appl. 3 (1958), 101-105.

10. S. Minakshisundaram, On the roots of a continuous non-differentiable function, J. Indian Math. Soc. 4 (1940), 31-33.

11. M. Morayne, On continuity of symmetric restrictions of Borel functions, Proc. Amer. Math Soc. 93 (1985), 440-442.

12. K. Padmavally, On the roots of equation $f(x)=\xi$ where $f(x)$ is real and continuous in $(a, b)$ but monotonic in no subinterval of $(a, b)$, Proc. Amer. Math. Soc. 4 (1953), 839-841.

13. G. Petruska and M. Laczkovich, Baire 1 functions, approximately continuous functions, and derivatives, Acta Math. Hungar. 25, (1974), 189-212.

14. S. Saks, Theory of the integral, 2nd Rev. Ed., Dover Publications, New York, 1964.

15. W. Sierpinski and A. Zygmund, Sur un fonction qui est discontinue sur tout ensemble de puissance du continu, Fund. Math. 4 (1923), 316-318.

16. H. Whitney, Functions defined in closed sets, Trans. Amer. Math. Soc. 36 (1934), 63-89.

Division of Mathematics, Auburn University, Auburn University, Alabama, 36849-5310 